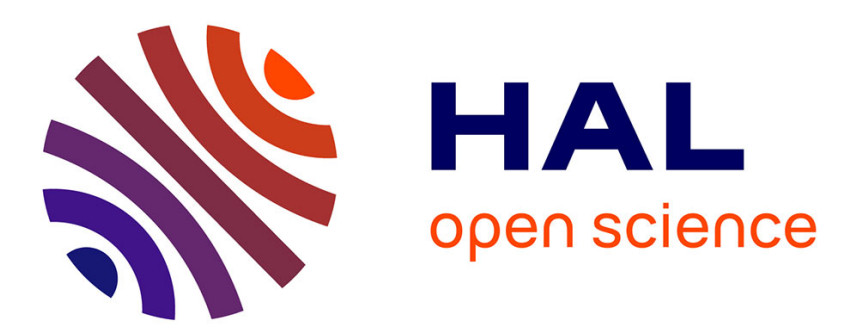

\title{
New Automatic and Robust Measures to Evaluate Hearing Loss and Tinnitus in Preclinical Models
}

\author{
A. Laboulais, S. Malmström, C. Dejean, M. Cardoso, T. Le Meur, L. Almeida, \\ C. Goze-Bac, S. Pucheu
}

\section{- To cite this version:}

A. Laboulais, S. Malmström, C. Dejean, M. Cardoso, T. Le Meur, et al.. New Automatic and Robust Measures to Evaluate Hearing Loss and Tinnitus in Preclinical Models. New Therapies to Prevent or Cure Auditory Disorders, Springer International Publishing, pp.159-186, 2020, 10.1007/978-3-03040413-0_7 . hal-03450469

\section{HAL Id: hal-03450469 \\ https://hal.science/hal-03450469}

Submitted on 26 Nov 2021

HAL is a multi-disciplinary open access archive for the deposit and dissemination of scientific research documents, whether they are published or not. The documents may come from teaching and research institutions in France or abroad, or from public or private research centers.
L'archive ouverte pluridisciplinaire HAL, est destinée au dépôt et à la diffusion de documents scientifiques de niveau recherche, publiés ou non, émanant des établissements d'enseignement et de recherche français ou étrangers, des laboratoires publics ou privés. 


\title{
New Automatic and Robust Measures to Evaluate Hearing Loss and Tinnitus in Preclinical Models
}

Laboulais $A^{12}$, Malmström $S^{2}$, Dejean $C^{2}$, Cardoso $M^{1}$, Le Meur $T^{3}$, Almeida L ${ }^{3}$, Goze-Bac C ${ }^{1}$, Pucheu $\mathrm{S2}^{1}$

\author{
1 Charles Coulomb Laboratory (L2C-BioNanoNMRI team), UMR 5221 Centre National de la Recherche Scientifique \\ - University, Montpellier, France \\ 2 CILcare, Advanced solution for drug development in hearing disorder, Montpellier, France \\ 3 Keeneye, Paris, France
}

Correspondence should be addressed to Pucheu Sylvie: sylvie.pucheu@cilcare.com

Keywords: Cochleogram, automated method, rat, hearing loss, tinnitus, MEMRI

\begin{abstract}
A. Laboulais
Charles Coulomb Laboratory (L2C-BioNanoNMRI Team), UMR 5221 Centre National de la Recherche Scientifique-University, Montpellier, France

CILcare, Advanced Solution for Drug Development in Hearing Disorder, Montpellier, France

S. Malmström · C. Dejean · S. Pucheu (*)

CILcare, Advanced Solution for Drug Development in Hearing Disorder, Montpellier, France e-mail: sylvie.pucheu@cilcare.com

M. Cardoso - C. Goze-Bac

Charles Coulomb Laboratory (L2C-BioNanoNMRI Team), UMR 5221 Centre National de la Recherche Scientifique-University, Montpellier, France
\end{abstract}

T. Le Meur · L. Almeida

Keeneye, Paris, France 


$\begin{array}{ll}\text { Abbreviations } \\ \text { OHC } & \text { Outer hair cells } \\ \text { IHC } & \text { Inner hair cells } \\ \text { SGNs } & \text { Spiral ganglion neurons } \\ \text { HCs } & \text { Hair cells } \\ \text { IC } & \text { Inferior Colliculus } \\ \text { FDA } & \text { US Food and Drug Administration } \\ \text { BBN } & \text { Broad-Band-Noise } \\ \text { BOLD } & \text { Blood oxygen level dependent } \\ \text { CC } & \text { Cerebral Cortex } \\ \text { fMRI } & \text { Functional Magnetic Resonance Imaging } \\ \text { GPIAS } & \text { GAP inhibition of the acoustic startle reflex } \\ \text { IP } & \text { Intraperitoneal } \\ \text { MEMRI } & \text { Manganese Enhancement Magnetic Resonance Magnetic } \\ \text { MnC12 } & \text { Manganese Chloride } \\ \text { NMR } & \text { Nuclear Magnetic Resonance } \\ \text { PET } & \text { Positron Emission Tomography } \\ \text { rCBF } & \text { Regional cerebral blood flow } \\ \text { R2 } & \text { NMR relaxation rate } \\ r_{i} & \text { Relaxivity } \\ \text { RGB } & \text { Red Green Blue } \\ \text { ROI } & \text { Region of Interest } \\ \text { SCs } & \text { Supporting cells } \\ \text { SEM } & \text { Standard Error of the Mean } \\ \text { SIR } & \text { Signal Intensity Ratio } \\ \text { SIT } & \text { Salicylate-induced tinnitus } \\ \text { SNR } & \text { Signal to Noise Ratio } \\ \text { T2 } & \text { Transversal relaxation time } \\ \text { TT } & \text { Trans-tympanic } \\ & \end{array}$




\begin{abstract}
During this collaboration between CILcare and KeenEye Technologies, a full pipeline has been designed to automatically classify and quantify the number of hair cells in 3D cochlea images. This project introduced many challenges with regards to specific pre- and post-data processing and an adaptive model for 3D object detection. The model has been trained using transfer learning with mini batch images keeping the context information around the different types of cells. This new automatic counting method performed 10 times faster than humans, with on average 3.5 minutes to analyze one fragment image. The algorithm gave performance metrics of $90 \%$ for precision and $70 \%$ for sensitivity. While the precision value is good, additional work is needed to increase the overall sensitivity and reduce its variance. In addition, an objective quantification method to detect tinnitus on rats was developed in collaboration between CILcare and Charles Coulomb Laboratory (L2C-BioNanoNMRI team). Tinnitus, a phantom auditory sensation which occurs in the absence of an external sound stimulus, is generated presumably within the auditory brain. Here we focus on the inferior colliculus (IC), a midbrain structure that integrates auditory information from both ears as well as information from other sensory systems. Some studies reveal neural hyperactivity in the IC after salicylate drug administration. In this study we present an innovative manganeseenhanced magnetic resonance imaging (MEMRI) analysis method called $\Delta R 2 / R 2$. This quantitative method detects $1 \mathrm{H}$ NMR relaxation rate changes in the absence or presence of tinnitus. The $\Delta \mathrm{R} 2 / \mathrm{R} 2$ method generates relevant data comparable to those obtained with the Signal to Noise Ratio (SNR) and Signal Intensity Ratio (SIR) methods when manganese is administered by the trans-tympanic or intraperitoneal route. A major advantage of the $\Delta R 2 / R 2$ method is that it is automatic, robust and reveals quantitative markers compared to qualitative methods like SNR and SIR.
\end{abstract}




\section{INTRODUCTION}

It is estimated that more than 500 million persons worldwide suffer from hearing disorders, which can be due to many different factors such as acoustic trauma, aging or drug toxicity [1,2]. Hearing loss, the most common sensory handicap, occurs when structures in the inner ear (the stria vascularis, outer hair cells (OHC) or inner hair cells (IHC) in the organ of Corti or spiral ganglion neurons (SGNs) are damaged [1]. The most widely used techniques for assessing preclinical hearing and hearing impairments are functional electrophysiological (evoked potential) and electro-acoustic (distortion products) measurements, or morphological observations that involve histological examinations or cell counting from tissues in the cochlea or brain. One of the most common histological measure is the cochleogram which consists of evaluating and counting hair cells (HC). The first difficulty of the cochleogram technique lies in obtaining a preparation of good quality, ideally one that encompasses the entire length of the basilar membrane from the highfrequency base to the low-frequency apex. The second difficulty is making accurate counts of HC, both those that are considered present as well as those that are absent. The counting procedure is typically performed manually or in some cases with a semi-automated method. The manual counting procedure requires much attention and expertise, is time consuming and potentially subject to observer bias. These limitations make manual, observer-based counts problematic for use in preclinical research and the development of new therapeutic solutions.

Tinnitus, another major hearing disorder that can be disabling, is a phantom auditory sensation which occurs in the absence of an external sound stimulus. Currently, one third of the population experiences tinnitus at least once in their life and 1-5\% develop persistent tinnitus with serious complications [3]. One major obstacle for detecting tinnitus in animal models arises from the fact that tinnitus is a subjective phenomenon. In humans, subjective tinnitus characteristics and severity can be obtained through questionnaires and self-reports [4]. In animal models, researchers have developed various behavioral techniques to evaluate tinnitus [5]. However, many of these behavioral models are time-consuming because they require a lot of animal training and seldom give quantitative results related to the loudness, pitch or severity of the tinnitus percept. To circumvent these difficulties, the scientific community is developing in parallel neuro-imaging techniques to observe neural signatures of auditory activities. The first utilization of the manganese enhanced magnetic resonance imaging (MEMRI) for the detection of tinnitus in animal models was reported in 2005 [6-8]. Nevertheless, more robust and standardized MEMRI acquisition protocols and analysis methods need to be established. Indeed, there are no standard tools available for automated procedures in tinnitus analyses. The following chapter presents recent developments in these research areas, aiming to develop new automatic techniques that are efficient and wellcontrolled to produce cochleogram to detect hearing loss and robust procedures to quantify by MEMRI the presence of tinnitus in animal models. 


\section{EVALUATION OF HUMAN AND COMPUTER-ASSISTED QUANTIFICATION OF HAIR CELLS IN FULL-LENGTH COCHLEA}

\subsection{INTRODUCTION}

The relationship between cochlear $\mathrm{HC}$ functional readouts and actual $\mathrm{HC}$ damage or loss after cochlear injury is complex and not straightforward [9-11]. Nevertheless, in preclinical research the cochleogram is fundamental tool used to quantify $\mathrm{HC}$ damage, evaluate treatments aimed at limiting the loss of $\mathrm{HC}$ or testing methods for inducing their $\mathrm{HC}$ regeneration. Moreover, in pharmacological safety and the evaluation of ototoxicity of new or re-positioned drugs, the cochleogram is one of the assays required by the FDA [12].

The cochleogram technique has been widely used for decades by academic researchers, but with no standardization of techniques or counting procedures [13]. These authors suggested a standardization of the cochleogram procedure stating that: (i) basilar membrane length should be plotted as a percentage instead of in millimeters, due to the biological variations that exists in basilar membrane length within a particular species and strain, and the total length in millimeters stated on the cochleogram; (ii) the equations used for frequency-place maps should be stated on the cochleogram.

One of the difficulties of the cochleogram technique lies in obtaining a good quality preparation of the sensory epithelium containing the organ of Corti and its rows of HC. In humans, the cochlea is located in the temporal bone, the hardest bone in the body. Two major categories of tissue preparation are commonly used to evaluate the sensory epithelium, either embedding and sectioning, or careful dissection of the sensory epithelium as a flat surface (or whole mount) preparations [14]. In both methods, the HCs can be labeled with different staining protocols, histological stains, fluorescent markers or immunolabeling methods, and visualized using a variety of microscopic techniques. The preparation consist of embedding the cochlea in a medium, usually paraffin or plastic, and then serially sectioning it to expose the hair cells [14-16]. The sections can be either parallel or perpendicular to the modiolar axis.

For the flat surface preparation method, the organ of Corti is extracted from the cochlea by microdissection into cochlear turns or half-turns. The cochlear fragments are prepared as flatly as possible by trimming off surrounding structures and the mounting the specimens on a microscope slide. The labeled hair cells are then visualized for counting along the entire length of the organ of Corti $[13,14]$. For the assessment of HC survival and construction of a cochleogram, the flat surface method is often preferred, due to its relative simplicity, comprehensive assessment over the length of the cochlea and the speed of cell counting; moreover, immunohistochemistry can be more easily performed on dissected tissue than on plastic embedded sections [14]. 
The HC counting procedure is typically performed manually or with a semi-automated method. In mouse cochleae, there are $\sim 700$ IHCs and $\sim 2400$ OHCs $[17,18]$ and in rat cochleae from the Sprague-Dawley strain there are $\sim 1000$ IHCs and 3700 OHCs [19].

In the guinea pig cochleae, there are approximately 3 times more HCs than in the mouse and 2 times more than in the rat ( $2000 \mathrm{IHCs}$ and $~ 7000$ OHCs) [20]. The manual counting procedure requires much attention and expertise and is very time-consuming. The loss of cochlear hair cells has been widely used in various experimental animal models, including guinea-pigs, chinchillas, rats, and mice [21-25].

In the field of preclinical development of new therapies, there is great interest to develop new automatic techniques for counting hair cells that are rapid, efficient, unbiased and well-controlled. In this context, we proceeded to develop an algorithm capable of automatically classifying and counting HCs in cochlear fragment images from flat surface preparations. To validate and determine its accuracy, the automatic results were compared to the results from manual counting performed by several experts. Many characteristics inherent to the input data, such as the type of image, the morphologic characteristics of the objects or unknown user accuracy are required to develop a specific pipeline for this project.

\subsection{MATERIAL AND METHODS}

\section{Generation of Data}

Cochleae were sampled from 3 control and 3 cisplatin treated Wistar rats $(10 \mathrm{mg} / \mathrm{kg}$, single IP injection). Three days after cisplatin treatment, the cochleae were removed and immediately fixed in 4\% PFA and then decalcified in 10\% EDTA for several days. The membranous and sensory spiral containing the organ of Corti was dissected using a flat surface preparation yielding 5 - 6 fragments per cochlea. The hair cells in each fragment were immunolabeled using a primary antibody against Myosin-VIIa, and a fluorescent secondary antibody (protocol adapted from Akil and Lustig [21]). The fragments were then mounted on a microscope slide and images acquired on a confocal microscope (20X objective). Each $x-y$ image plane contained approximately $2000 \mathrm{x}$ 3000 pixels, with a two-dimensional resolution of 0.642 microns per pixel for each focal plane. Approximately 30 focal planes were obtained per fragment, each plane separated by 1.64 microns.

Three independent scientists, highly experienced in cochlear cell counting, independently manually counted and annotated all IHCs and OHCs in each cochlear fragment in 6 cochleae (3 control and 3 cisplatin). Users were instructed to annotate every labeled cell with a point annotation within the cell's 3-dimensional center and identify them either as an IHC or OHC. For the $\mathrm{OHCs}$, their respective row location $(1,2$ or 3$)$ was taken into account. Users were blinded to the treatments. Fragments were identified by a unique number that specified both the cochlea and fragment number. 
The labeled point-annotation was obtained for each cochlear fragment, with the exception of fragments in which the user could not identify any cells as being present. Majority voting (two out of three) based on the value for each cell was used to determine a unique set of point annotations for each fragment.

\section{Algorithm development}

Due to the volumetric nature of the image data, together with the fact that users annotated the cells using a 2D stack viewer, we developed a 3D object detection algorithm that would perform detections on each stack. A model based on a deep convolutional neural network (CNN) was first used in order to get the detections for each stack of 3D images [26] The last feature map of the CNN allowed us to predict the cell's locations and class (IHC or OHC). Predictions were then grouped using an unsupervised method to merge two dimensional predictions into a subset of unique 3D hair cell detection specification.

The image's characteristics directly impact the development of the algorithm. The difference between the pixel size along the depth (between each stack) and the pixel size on the 2D x-y plan must be carefully taken into account in order to provide consistent detections. The whole 3D images cannot be loaded and analyzed at once by the network for both training and routine use due to their excessive sizes. Pre-processing the images before using them in the algorithm is therefore a necessity. In addition, the image quality can vary depending on several parameters such as the treatment of the animals or if they originate from healthy controls or cochleae damaged by noise or ototoxic agents. The fragment location within the cochlea can also affect the quality of the final image. The first fragments from apical or middle regions of the cochlea tend to be less damaged than fragments from the basal regions, where damage due to dissection, preparation or treatment are more likely to occur. In these cases, the context information for the cell detection is degraded or completely lost due to extensive damage.

As previously discussed, the characteristics of the objects are contained in their relative locations in the 3-dimensional volume and their morphology is revealed by the staining procedures. All hair HCs are small relative to the size of the image and share large similarities between the two classes (i.e., IHCs vs OHCs). In order to perform proper classification, the algorithm needs to be aware of a large context area surrounding each object, because the context area provides important criteria for differentiating the different cell types. Moreover, OHCs and IHCs are not equally distributed within the dataset because there are approximately three times more OHC than IHC per fragment image. The unbalanced dataset makes the learning process of the network more difficult for the under-represented objects. 
In order to train the 3D deep convolutional object detection network, we used five different fragments from two separate cochleae, one control and one cisplatin treated. Each fragment is comprised of a stack of thirty-five slices of various dimensions (approximately 3000 x 2000 pixels). To limit bandwidth during training, each 3D image was subdivided into smaller images. However, the subregion was sufficiently large that it included both IHCs and OHCs. These subregions covered an area of $320 \mu \mathrm{m}$ x $320 \mu \mathrm{m}$. Each expert only annotated the cell's center with a point in order to reduce the annotation time and have a sufficient amount of data for the training process. Using the average size of the cells, the point annotations are added to 3D box annotations. Those last annotations are then used by the network as localization ground truth (GT) information, i.e., the position and size that should be inferred. The overall dataset was partitioned in two sub datasets.

Two cochleae containing $90 \%$ of the subregions were used for training and cross validation while $10 \%$ was set aside for evaluation of statistical metrics. Then, 4 separate cochleae were used for full cochleogram comparisons. To increase the number of examples without increasing the amount of initial data, image augmentations such as rotations, scaling and contrast manipulations were performed on the training set in order to further train the model.

Transfer learning is a widely used technique in deep learning was used to provide a basic knowledge to the network before training saving time and data [27]. The convolutional neural network is initialized with another network pre-trained on a similar task. During the training session, the model parameters are optimized to reduce the error of cell localization and classification with regards to the ground truth annotations. This optimization is done with a gradient descent algorithm called stochastic gradient descent (SGD) with momentum [28]. While accumulating error information from the previous predictions, it enables training with only one 3D image at a time without instability.

In order to assess the performance of the algorithm, a comparison was performed between manual and automated counting of hair cells. Two cochleograms with IHC and OHC-were computed from the two sets of annotations, one evaluated by humans and one evaluated algorithmically using a line drawn by human experts along the IHCs of each cochlear fragment. The line length for each fragment was converted into a percentage regarding the total cochlear length and the density of cells was computed for each 5\% interval. The average and standard deviation (SD) for hair cell density were then calculated and plotted for each percent distance. 


\subsection{RESULTS}

\section{Algorithm Development Results}

The quality of the training was evaluated with a Precision-Sensitivity curve for each class. The Precision-Sensitivity curve, also called Precision-Recall curve, summarizes the trade-offs between the true positive rate (sensitivity) and the precision of the model using different probability thresholds. For each probability, the number of true positive detections is defined using a specific matching method. A distance threshold vector, representing the average semi-axis values of a cell along each dimension is used to define whether or not a detection matches a ground-truth annotation. Figure 1 shows the Precision-Sensitivity curves for IHC and OHC objects. The IHC curve shows a drop of precision for low sensitivity due to cells correctly detected but counted as false positive because it was not annotated by the expert. The two optimal points of these curves (red dots in Figure 1) were then used to get the optimal probability threshold for each class. To provide quality results when running the algorithm on unknown fragments, cell detections were filtered with those thresholds regarding their confidence probability outputted by the network.

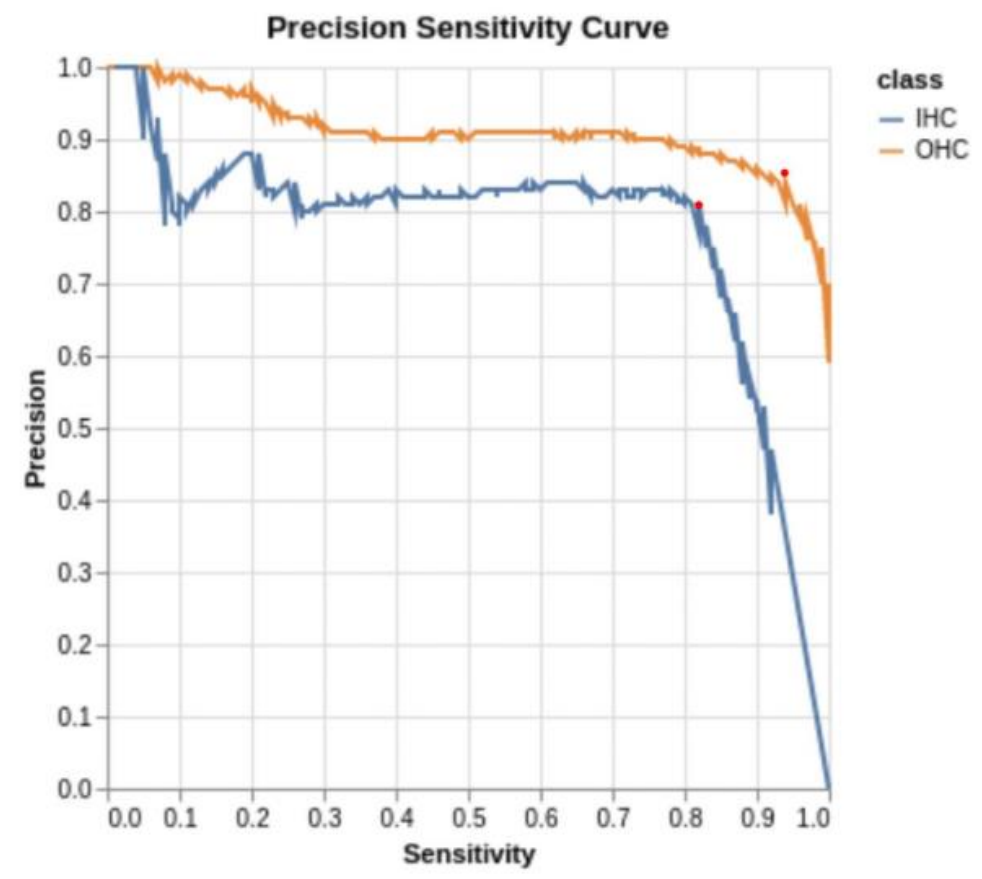

Fig. 1 Precision-sensitivity curves for IHC and OHC objects on the test subregions. Note the lower overall performance for IHCs is due to the bias of over sampling of OHCs 


\section{Comparison of counting time}

One of the main advantages of an algorithmic approach is speed, reliability, and reproducibility. Regarding time, each user took on average $30.6 \pm 6.5$ minutes to fully annotate a fragment containing on average $887 \pm 125$ cells (Figure 2). The algorithm, run through the KeenEye Platform, spent $3.5 \pm 1.2$ minutes to finalize the same analysis, showing a $\sim 10$-fold gain of time. This time represents the incompressible time for the algorithm to detect the hair cells, but also the data preparation and the post processing done in order to display the detection on the platform. For the automatic analysis, the small variation of duration on the fragment images is highly related to the size of the image; the larger the image, the longer the counting time.

\section{Quality of Hair Cell Counting}

The difficulty of the counting task can be illustrated by the variability of the IHC and OHC counts among the different experts. The count variability among the experts, measured as the standard deviation of counts over the mean of counts per fragment, was (median \pm SD) $8.9 \pm 13 \%$ for IHCs and $18.3 \pm 33 \%$ for OHCs counts (Figure 3).

The algorithm performance measures were quantitatively measured on the test set with two metrics: precision and sensitivity. The precision represents the percentage of correct results, while the sensitivity refers to the percentage of ground truth object correctly detected. The fully automated detection and counting software achieved a precision of $89 \pm 9 \%$ and sensitivity of 69 $\pm 12 \%$, the relative distributions are shown in the box-whisker diagrams in Figure 4 . The high precision of the algorithm ensures that each one of its detections is close to a ground truth annotation; however, this lower the sensitivity and increases the variability. This result is explained by the difficulties the algorithm has performing correct cell detection on damaged fragments where the context information is missing or corrupted.

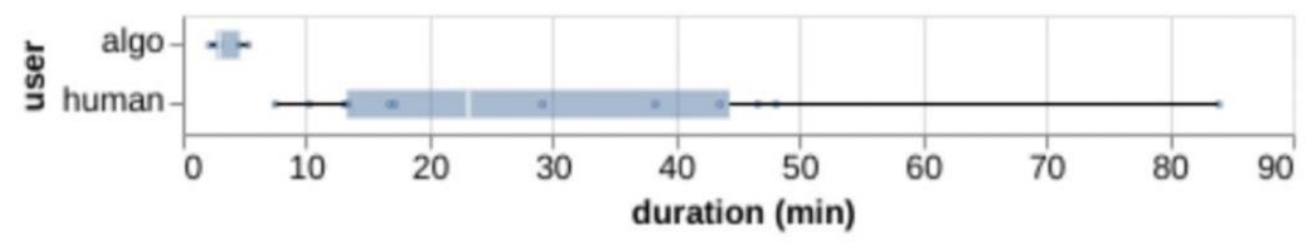

Fig. 2 Time in minutes for annotating each fragment among human users compared to the algorithm 


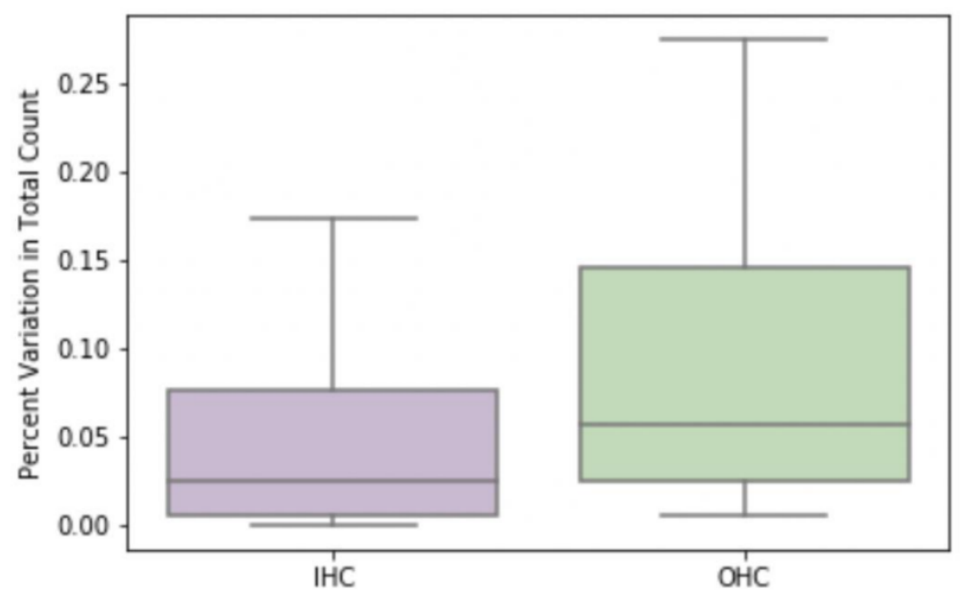

Fig. 3 Box-Whisker (median \pm SD) distributions for $\%$ variability of counts for manual counting

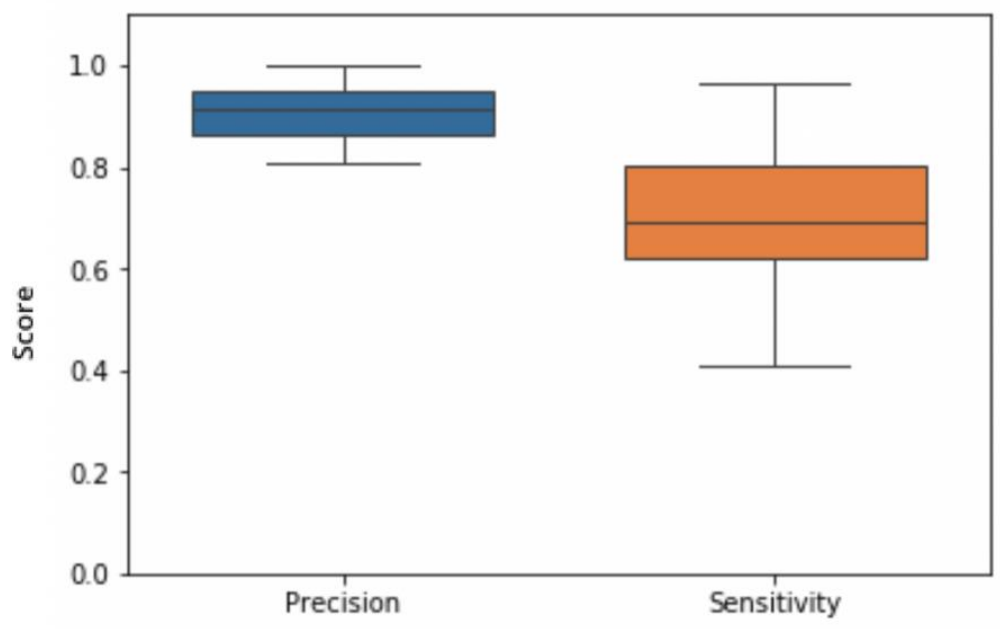

Fig. 4 Box-Whisker plots (median \pm SD) for the sensitivity and precision scores for the algorithm versus test cases

\section{Cochleogram analysis}

The cochleogram analysis was performed on cisplatin-treated and control cochleae. Using a line drawn along the IHCs by an expert, we computed the full cochleograms resulting from the manual and automated counting and compared them to each other (Figure 5). For this dataset, manual and automated counting procedures had similar standard deviations. Furthermore, the differences among cochleograms were not significant, with significance determined by a Mann-Whitney test comparing the distribution of differences between the human and automatic counting at each bin. 
Using the closest point on this line to any detection, we computed cell density cochleograms. Once the cell count along this line was grouped for all fragments in a cochlea, counts were binned along the length of the entire cochlea. The size of the bins was chosen to be $5 \%$ of the total cochlea length, and thus varied among cochleae. The average and SD for the hair cell density were then calculated and plotted for each percent distance. The results are shown in Figure 5, where we compared all expert annotations (denoted as human) to the algorithm results; this allowed us to see the variation in counts due to expert disagreement along the cochlea. Both counting methods resulted in a cochleogram with similar profiles all along the cochlea for IHCs and OHCs. The IHC density curves in the two groups were essentially the same with both manual and automated counting methods and close to $0.1 \mathrm{cell} / \mu \mathrm{M}$. Concerning the OHCs of the cisplatin-treated cochleae, a lower sensitivity was noted in the apical half of the cochlea with automated counting while in the basal half both methods gave similar curves. For the control group, a difference in OHC density between the manual and automated counting procedures was observed all along the cochlea.

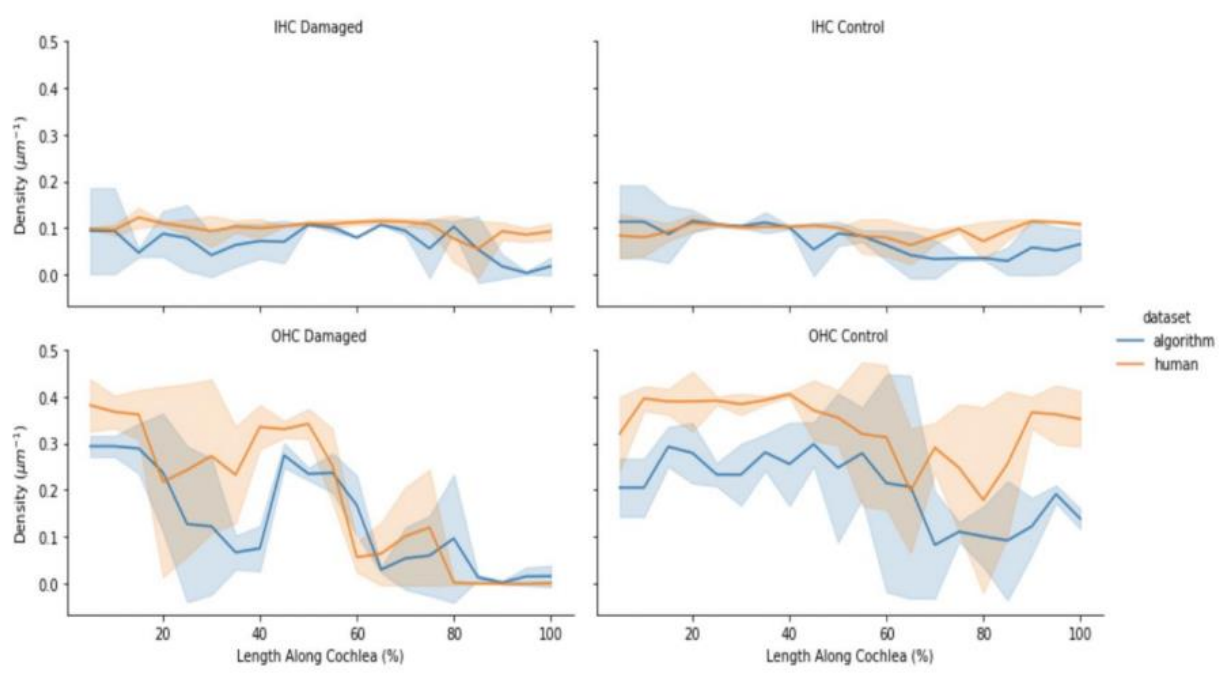

Fig. 5 Example comparing cochleograms obtained from human manual (orange) counting and automated (blue) counting of OHCs and ICHs in cisplatin-treated rats. Solid line is the mean with bands for the standard deviation

\subsection{DISCUSSION}

Many studies rely on cochleogram analyses, but most of them use manual cell counting. A common procedure is to manually count the present and absent hair cells directly under the microscope using an eyepiece to measure and segment the basilar membrane. Hair cell loss is then plotted as a function of the distance from apex, either in $\mathrm{mm}$ or in length converted to \% [13, 29]. A stereological approach has also been used on phalloidin-stained stereocilia bundles as counting units [30]. 
During this collaboration between CILcare and KeenEye Technologies, a full pipeline has been designed to automatically classify and quantify the number of hair cells in 3D cochlea confocal images based on flat surface preparation and fluorescent labeling of hair cells. This project introduces many challenges with regards to specific pre- and post-data processing, and an adaptive model for 3D object detection. The model has been trained using transfer learning with mini batch images keeping the context information around the different types of cells. This new automatic counting method was 10 times faster than the human method, taking on average 3.5 minutes to analyze one fragment image. Indeed, even a trained expert performing manual counting of all labeled hair cells in a cochlea requires a minimal time to register the cell count, with a mouse click or a manual cell counter. Alternative methods exist to produce a cochleogram where only the missing cells and the length of the fragments are counted and the normal (ground truth) cell density is used to get the total number of cells. This method is relatively efficient for cochleae with little cell loss or a high degree of cell loss, but for intermediate cell loss the counting procedure is longer.

It is important to note that due to the possible non-specificity of the staining, together with the fact that a single stain was used, cell identity is often determined using subtle information associated with the cells relative to their positions and morphology. For example, OHCs are grouped in 3 parallel rows while the IHCs form a single row. Occasionally, extra OHCs may be observed radial to the third $\mathrm{OHC}$ row anywhere along the length of the organ of Corti. The organization of the $\mathrm{OHCs}$ is generally less ordered at the apical end than at the base or in the middle regions and is also dependent on possible organ health and/or damage. Cell identification becomes extremely subjective when the cochleae are highly damaged, or staining is incomplete, making unambiguous identification almost impossible. This ambiguity is explicitly shown in Figure 6, where only a single IHC row has been annotated by only one expert.

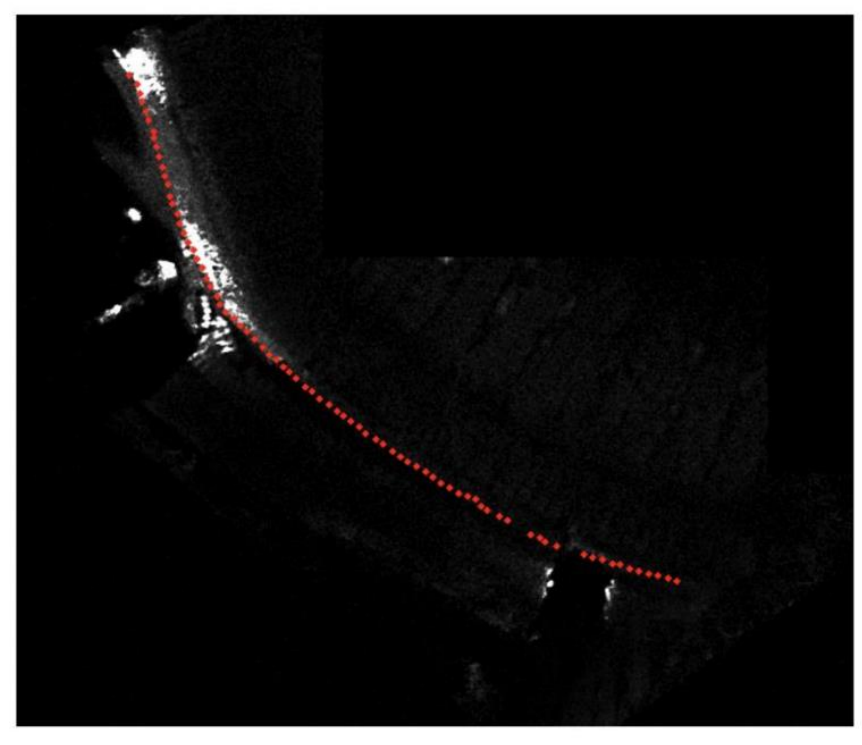

Fig. 6 Image of cochlear fragment where only a single row of IHCs (red dots) is annotated 
The algorithm gave performance metrics of $90 \%$ for precision and $70 \%$ for sensitivity. While the precision is good, improvements are needed to increase the overall sensitivity, while reducing the variance. Developing an end-to-end 3D convolutional neural network to capture the 3D features of each cell could improve the performance of the algorithm making it more robust for analyzing images of different quality. However, this technique requires more computational memory resources and is unable to use large context information for cell classification. In order to improve detection results, object balancing could be added to the data preprocessing step before the training paradigm. Methods such as weighting the error for each detection regarding its ground truth class, or under-represented object augmentation can be used to solve this problem. However, the impact of these methods on the overall metrics has not been established. The algorithm already performs properly on IHC detection due to greater context information awareness where the IHCs are anatomically more isolated and thus their identification is less ambiguous. At this stage, algorithm performance is highly correlated with the state of the fragments in the images.

Fragments can be damaged during dissection, leading to images where cell integrity is not well preserved. The model was trained on images of good quality; however, the model performance decreases on damaged fragments as the context information is corrupted.

Detection results on damaged images, and on the overall set of cochlear images, could be improved by adding damaged samples to the training dataset.

This algorithm, and the full pipeline associated with it, can be re-used for future projects. The model can conserve its performances on images with small variations of characteristics regarding the initial fragment images it was trained on. This would be true for hair cell counting projects with similar cochlear images but coming from different species. However, if the image acquisition changes, it might cause important changes in the characteristics of the input images (e.g., number of channels, type of labeling, etc.). In this case, this algorithm can still be used via transfer learning, providing a knowledge base for a future one. The new training will be faster and will require a smaller amount of data to reach similar performances. 


\section{COMPARISON OF THREE METHODS TO ANALYZE PRECLINICAL MANGANESE ENHANCED MAGNETIC RESONANCE IMAGING ON SALICYLATE-INDUCED TINNITUS IN RAT MODELS}

\subsection{INTRODUCTION}

The phantom sound of tinnitus is believed to be generated at certain points in the auditory system; it is generally triggered/initiated by cochlea damage, resulting in neural changes perceived as a phantom auditory sensation in the auditory cortex or other parts of the central nervous system. It can be triggered by noise-induced hearing loss, presbycusis, otosclerosis, otitis, Meniere's disease, or by ototoxic medications [31]. Tinnitus models in animals are mainly based on salicylate-induced tinnitus and noise-induced tinnitus. One major obstacle for the detection of tinnitus in these models arises from the fact that tinnitus is a subjective phenomenon, the only possible diagnosis relies on self-reports of the subjects in humans [4]. In animals, researchers have developed behavioral models using basic mechanisms of conditioning to evaluate tinnitus. It was first demonstrated by Jastreboff and colleagues [32] and later several extensions of that work have been published [5, 33]. Over the last few years, the GAP inhibition of the acoustic startle reflex (GPIAS) [34], has increasingly been used. GPIAS uses the amplitude of the acoustic startle reflex to a noise burst as a behavioral readout. The startle-evoking noise burst is presented on a background of moderateintensity continuous noise. A silent interval, or gap, which serves as a pre-pulse, is presented shortly before the high-intensity noise burst. If a normal hearing animal hears the silent gap preceding the high-intensity noise burst, the startle reflex amplitude is inhibited. However, if an animal has tinnitus, the salience of the silent interval is reduced presumably because the phantom sound of tinnitus "fills in" the gap. In cases where the gap stimulus fails to significantly inhibit the amplitude of the startle reflex, the animal is presumed to have tinnitus. The frequency, bandwidth and intensity of the carrier signal in which the gap is embedded can be varied with a goal of estimating the pitch and loudness of the tinnitus [35]. The GPIAS method does not require animal training. However, the methods used to collect and analyze GPIAS data varies between laboratories making the comparison of results and reproducibility of data difficult [36]. In parallel, the auditory scientific community has become increasingly interested in developing imaging tools to detect tinnitus in animals. Indeed, neuroimaging techniques enable one to non-invasively visualize and study in vivo brain activities. Nowadays, the neuroimaging methods used in animal models include such techniques as: manganese enhancement magnetic resonance Imaging (MEMRI), functional magnetic resonance Imaging (fMRI) and micro positron emission tomography (microPET). MEMRI and fMRI are based on nuclear magnetic resonance in strong magnetic fields to create various types of anatomical or functional brain images. MEMRI utilizes manganese chloride $\left(\mathrm{MnCl}_{2}\right)$ as a contrast agent to follow neuronal brain activity [37, 38].

$\mathrm{Mn}^{2+}$ ions enter active cells through voltage-gated calcium channels and accumulate inside neurons. 
Blood oxygen level dependent (BOLD) fMRI detects changes in deoxyhemoglobin concentrations under task-induced or spontaneous resting state conditions that reflect the degree of neural activity. PET imaging is based on the detection of radioactivity emitted by a tracer administered to the subject. With the appropriate tracer injected into blood circulation, it is possible to assess regional cerebral blood flow (rCBF) in the resting state or under task-based conditions.

These imaging methods can be compared in terms of advantages and drawbacks. In small animal imaging studies, high spatial resolution is essential because of the small size of the brain in rodents compared to humans. MEMRI can provide functional information with a resolution of about 100 $\mu \mathrm{m}$, whereas PET resolution is limited by the size of the gamma ray detectors, providing millimeter resolution. fMRI has a higher spatial resolution than PET, but lower resolution compared to MEMRI (fMRI spatial resolution $\sim 500 \mu \mathrm{m}$; MEMRI spatial resolution $\sim 100 \mu \mathrm{m}$ with high magnetic field strength. In addition, the contrast in MEMRI is more directly related to neural activity whereas fMRI relies on an indirect neurovascular coupling to obtain neuronal information. In addition, the anesthesia used to avoid animal movement in fMRI can affect the neuronal responses, including activity in the auditory system [39-42]. In contrast, $\mathrm{MnCl}_{2}$ is administered prior to imaging when the animals are awake, thereby avoiding the confounding effects of anesthesia. The utilization of MEMRI has been used since 2005 when it was discovered that the blood-brain barrier (BBB) did not need to be weakened in order for injected manganese to enter the brain and taken up into spontaneously active or stimulus-activated neurons [8]. Therefore, major auditory studies including tinnitus were carried out by using MEMRI in the past few years $[6,7,35,43,44]$. However, some tinnitus studies were performed with fMRI and PET on animal models $[9,45,46]$.

Currently there are controversies in the literature regarding the pathological basis of tinnitus, and the most appropriate analytical method to identify the neural signature of tinnitus. To accelerate the development of new therapies, it is essential to establish more robust and standardized MEMRI analysis methods. Here we present an innovative quantification technique based on magnetic resonance properties, which relies on the transverse relaxation rates, namely R2, which depends on the constituents of the tissues and the presence of contrast agent. The goal of this method is to precisely detect the accumulation of paramagnetic $\mathrm{Mn}^{2+}$ ions accumulated in active neurons through voltage-gated calcium channels [47]. The direct measure of the NMR signal in specific regions of the brain allows to determine the percentage change of R2 between auditory areas of interest versus non-auditory regions. A positive $\Delta \mathrm{R} 2 / \mathrm{R} 2$ indicates an increase of the nuclear magnetic resonance relaxation rate related to accumulation of $\mathrm{Mn}^{2+}$ ions in active neurons. In the following paragraph, the $\Delta \mathrm{R} 2 / \mathrm{R} 2$ quantification is compared with some other available MEMRI analysis methods, namely the Signal to Noise Ratio (SNR) and the Signal Intensity Ratio (SIR) [7, $35,44,48,49]$.

In this study, our analyses focused on a specific auditory structure, the inferior colliculus (IC). The IC plays important roles in processing auditory information received from both ears and then relaying the acoustic information to higher and lower brain regions [50]. 
The IC was also selected since several MEMRI studies reported an increase of neuronal activity in the inferior colliculus in animal models of tinnitus [7, 43, 48, 51]. The underlying hypothesis is that abnormal neuronal activity in the IC, as reflected in MEMRI, can provide a biomarker for the detection of tinnitus [6].

\subsection{MATERIAL AND METHODS}

\section{Animals (Figure 7)}

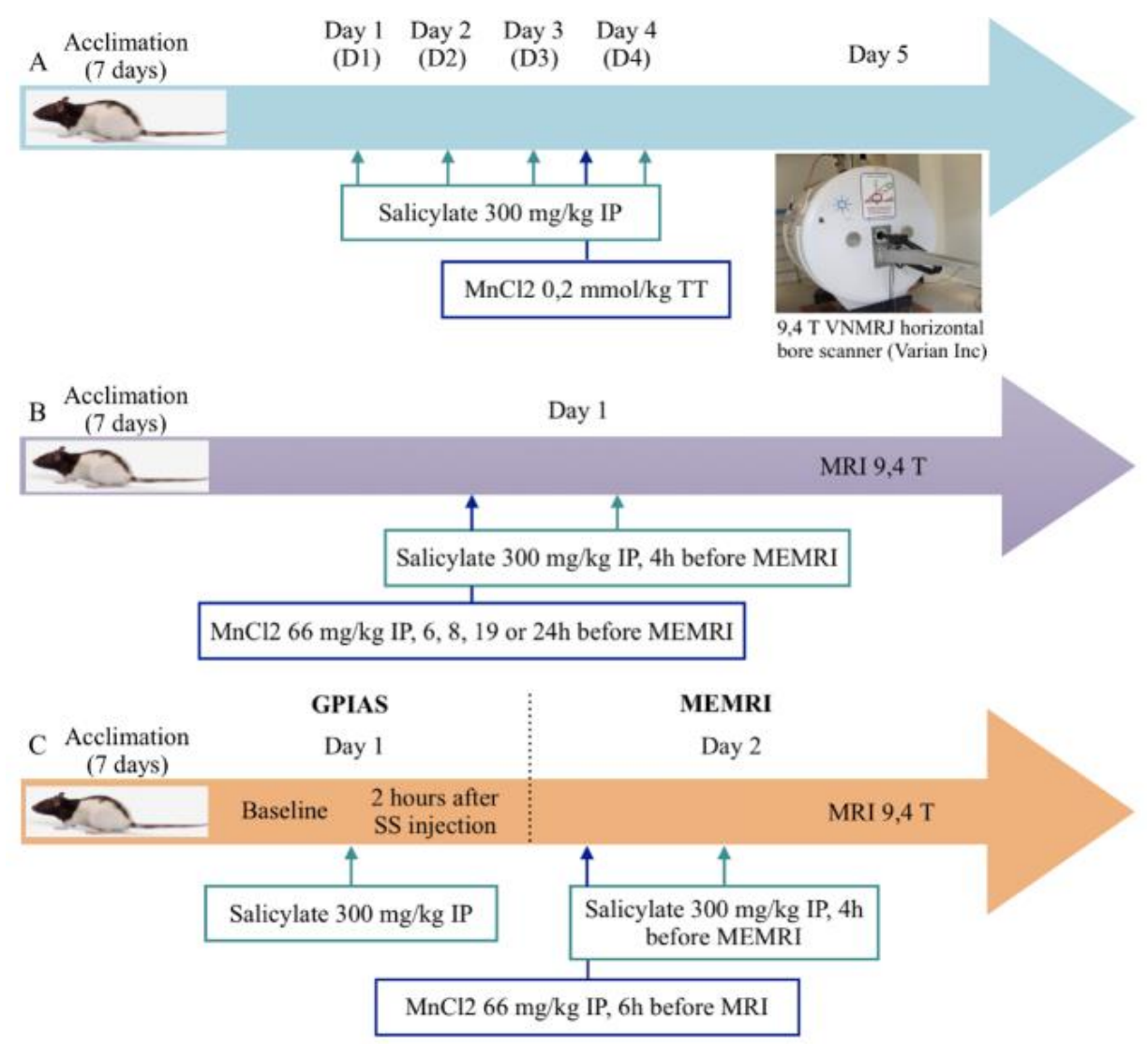

Fig. 7 Schematics of three different experimental protocols used in the study with $\mathrm{MnCl} 2$ in whichsalicylate was administered by (A) transtympanic administration $(\mathbf{A})$ and by $(\mathbf{B}, \mathbf{C})$ intraperitoneal route 
Forty male Long Evans rats ( 8 weeks old, weighting $250 \mathrm{~g}$ ) were maintained under specific pathogen-free conditions. Before starting the study, the rats were allowed to acclimate for 7 days in a colony maintained at $22 \pm 6{ }^{\circ} \mathrm{C}$ and a standard light/dark cycle. The animals were used in three experimental protocols: (1) The first series of animals (A) ( $\mathrm{n}=10,2$ groups) was injected with $\mathrm{MnCl}_{2}(0.2 \mathrm{mmol} / \mathrm{kg})$ via trans-tympanic administration [52] 24 hours before MEMRI measurement fourth day (D4); the salicylate-induce tinnitus (SIT) group received sodium salicylate (300 mg/kg, i.p.) once a day from the first day (D1) to the fourth (D4) and a similar volume of saline was injected into sham group once a day from first day (D1) to fourth (D4) (Figure 7) [3]. The second series (B) ( $\mathrm{n}=24,8$ groups) was injected with $\mathrm{MnCl}_{2}(66 \mathrm{mg} / \mathrm{kg}$ ) by an intraperitoneal route (i.p) at 6, 8, 19 and 24 hours before MEMRI measures. The SIT animals were treated with a single injection of sodium salicylate $(300 \mathrm{mg} / \mathrm{kg}$, i.p.) 4 hours before MEMRI measurement. The sham group was injected with a similar volume of saline 4 hours before MEMRI measurement. In the third series (C) ( $\mathrm{n}=6,2$ groups), GPIAS measurements were carried out in all animals, 2 hours after administration of salicylate $(300 \mathrm{mg} / \mathrm{kg}$, i.p.) and before the MEMRI protocol $\left(\mathrm{MnCl}_{2}\right.$, i.p. 6 hours, single injection of sodium salicylate, $300 \mathrm{mg} / \mathrm{kg}$, i.p, 4 hours before MEMRI measures) [3]. All animal experiments were conducted in accordance with European Directives (\#2010/63/UE).

\section{Salicylate}

Salicylate was dissolved in sterile physiological saline at $176.5 \mathrm{mg} / \mathrm{mL}$ and administered at 300 $\mathrm{mg} / \mathrm{kg}$ (i.p.). Sham salicylate animals received an injection of physiological saline.

\section{MEMRI acquisition imaging}

Before MRI image acquisition, rats were anesthetized using 2\% isoflurane and monitored (respiration and temperature) using the MR-compatible Small Animal Monitoring and Gating System (Model 1025, SA Instruments, Inc., New York, USA). Respiration was maintained around 40 breaths/min by adjusting isoflurane level and oxygen flow rate. During the MRI acquisitions, the animal is maintained in a stable position with the use of a heated cradle equipped with head

and tooth holder. The rat head is positioned at the center of the MRI magnet in a dedicated antenna fixed to the cradle.

MRI measurements were performed on a 400MHz MRI scanner (Agilent Varian 9.4/160/ASR, Santa Clara, California, USA) equipped with a MAGNEX TS1276D, a brain SHS coil $400 \mathrm{MHz}$ (BioNanoNMRI, France) associated with an imaging acquisition system (Agilent, Palo Alto, California, USA), anesthetic and animal holder systems from Minerve Siemens A.G., Erlangen, Germany)/ RS2D (Haguenau, France). 
Axial images were acquired using a T1-weighted MEMS (Multiple Echo Multi Slices) protocol with the following parameters: Field of view, $60 \times 30 \mathrm{~mm}$ matrix size, 512 × 256 axial slices with one zero filling, $1 \mathrm{~mm}$ slice thickness, no gap, $\mathrm{TR}=500 \mathrm{~ms}$, $\mathrm{TE}=12,63 \mathrm{~ms}$, number of averages $=8$, number of slices $=16$, scan time $=17 \mathrm{~m}$ and $45 \mathrm{~s}, \mathrm{NE}=2$.

\section{Gap inhibition of acoustic startle reflex}

Behavioral testing was conducted using Kinder Scientific startle reflex hardware and software customized for this application by the manufacturer. Gap detection testing with background sounds presented through one speaker and startle stimuli presented through a second speaker located in the ceiling of the testing chamber $15 \mathrm{~cm}$ above the animal's head. The floor of the chamber is attached to a force transducer which to measure the startle force applied to the floor. A clear polycarbonate animal holder, with holes cut for sound passage, was suspended above the floor allowing the animal to freely turn around while minimizing excessive movement. An adjustable-height roof was set to a level that keeps animals from rearing up, a behavior that adds variability to the startle response. Background sounds in the startle chamber consisted of $60 \mathrm{~dB}$ average SPL broadband noise (BBN) or bandpass filtered noise. Each test session began with a 3 min acclimation period followed by two trials consisting of a startle-eliciting noise burst (115 dB SPL, $50 \mathrm{~ms}$ duration); the acclimation period served to habituate the startle response to a more stable baseline. Data from the first initial trial was not used in the gap detection analysis. The remainder of the session consisted of startle-only trials presented before or after gap trials in a counter-balanced manner throughout the session. Gap trials were identical to startle-only trials, except for the inserted gap. The frequency of the background used to carry the gap was systematically varied throughout the remainder of the session. The background noise consisted of bandpass filtered noise bands at 4 frequencies $(10,12,16 \& 24 \mathrm{kHz})$ and also BBN presented with and without gaps. Stimuli were presented in ascending order from $10 \mathrm{kHz}$ to BBN for each test. The offset of the gap began $50 \mathrm{~ms}$ before the onset of the startle stimulus $(50 \mathrm{~ms}$ duration, $1 \mathrm{~ms}$ rise/fall time). In conditions where PPI is measured, prepulse stimuli match the backgrounds used in Gap detection in both spectral and intensity dimensions. PPI testing is essentially the inverse of Gap detection testing and serves as a valuable control for hearing loss and temporal deficits that might explain any Gap detection deficits. For example, rather than presenting a $60 \mathrm{~dB}, 10 \mathrm{kHz}$ signal in the background continuously and embedding a $50 \mathrm{~ms}$ Gap before startling the animal, during PPI testing the background is quiet and a $50 \mathrm{~ms}$ duration, $60 \mathrm{~dB}, 10 \mathrm{kHz}$ prepulse signal is presented before startling the animal. 


\section{Inclusion Phase of GPIAS groups}

Baseline auditory brainstem responses (ABR) were measured from both ears ( 8 animals by group) using tone burst presented at 2, 4, 8, 16 and $25 \mathrm{kHz}$; tone bursts intensity was varied from 90 to 0 $\mathrm{dB}$ SPL at each frequency. The measures were performed under isoflurane anesthesia. One animal with a threshold above $40 \mathrm{~dB}$ SPL in one ear at $16 \mathrm{kHz}$ was considered to have pre-existing hearing loss and was eliminated from the study. The animals selected were randomly distributed to the 6 groups ( 3 treatments groups each with an experimental and control group).

GPIAS testing ( $\mathrm{n}=6$ animals per group) was carried at 4 frequencies, 10, 12, 16 and $24 \mathrm{kHz}$ and BBN. Background sounds in the startle chamber consist of a 60 -dB peak SPL level broadbandnoise (BBN). Animals were acclimated to the apparatus for two consecutive days the week before the first GAP baseline measure was collected and baseline GPIAS measures were obtained. During baseline GPIAS tests, the amplitude of the startle reflex on trials containing the gap insertion (Gap) were expected to be lower than those without the gap (No Gap). Animals with putative tinnitus with features similar to the background sound fail to detect the silent gap, presumably due to their tinnitus.

Therefore, acoustic startle amplitudes on gap-trials were presumably similar to those without the gap (i.e., little or no inhibition of the startle reflex). For each animal, the calculation was realized from the median value (median \pm median absolute deviation) of the 12 trials (of fewer because of aberrant trials) for Gap detection test (No Gap, Gap). In addition, for each rat, the percentage of Gap inhibition ((1-Gap)/No gap) x 100 was calculated and the obtained values were average for each group (mean \pm SEM). As the startle occurred at $250 \mathrm{~ms}$, only pairs of trials of ((NoPPI/PPI) - NoGap/Gap) exhibiting a startle response within the time interval from 265 to $320 \mathrm{~ms}$ (i.e. 15 $\mathrm{ms}$ to $70 \mathrm{~ms}$ after startle stimulus) were included. 


\section{MEMRI analysis methods}

The MEMRI analysis is based on three different processing methods of the same set of MR images and regions of interest (ROIs), namely Signal to Noise Ratio (SNR), Signal Intensity Ratio (SIR) and the $(\Delta R 2 / R 2)$ methods. The SNR is defined as the mean MRI signal intensity in a given region divided by the image noise intensity [43, 44] (Figure 8).

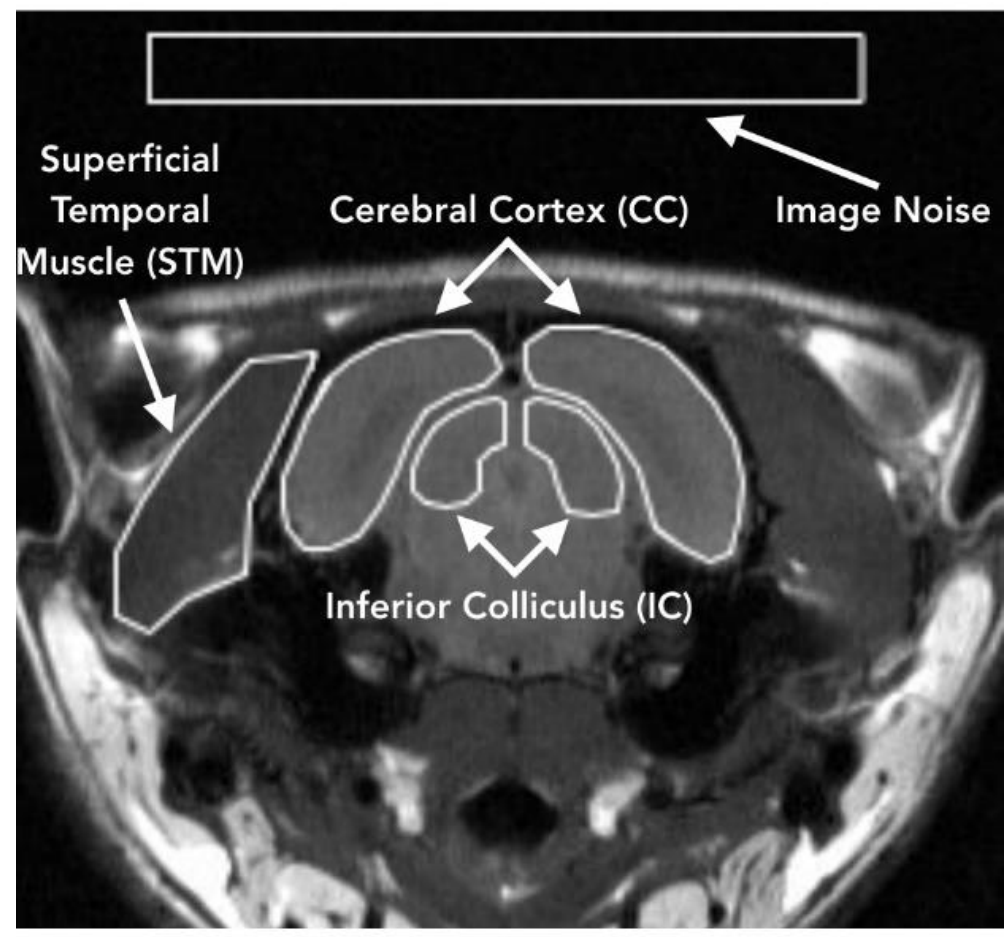

Fig. 8 T1-weighted MR coronal brain slice acquired with 9.4 T scanner (400 MHz). Arrows point to examined regions of interest (ROIs): Image noise, superficial temporal muscle (STM), inferior colliculus (auditory structure), and cerebral cortex (nonauditory structure). 
The SIR is defined as the mean MRI signal intensity in a given region of the brain divided by the mean MRI signal intensity in the superficial temporal muscle [7, 35, 48]. Finally, the ( $\Delta R 2 / R 2)$ method is based on the ratio enhancement of the relaxation rate $\mathrm{R} 2$ between two regions of interest the cerebral cortex (visual cortex, entorhinal cortex, subiculum and cingulum areas) and in the auditory structures (i.e., the IC). Figure 9 presents the procedures used for acquiring and processing the MR images and analyzing the results.

One axial brain slice that included the IC is selected. From a homebuilt program using a Matlab interface, the ROIs that included: (1) the superficial temporal muscle (STM), image noise intensity, [3] IC and (4) cerebral cortex (CC) are segmented as illustrated in Figure 8. The ROI segmentations of the IC and CC were carried out on both left and right sides of the brain with administrations of $\mathrm{MnCl}_{2}$ by the intraperitoneal and trans-tympanic ipsilateral side routes.

$\mathrm{R} 2$ relaxation rate maps can be calculated voxel by voxel in each ROI according to the following expression:

$$
R 2=\frac{1}{T E} \times \log \frac{e c h o 1}{e c h o 2}
$$

as presented using a Red, Green, Blue (RGB) color mapping in Figure 10.A and 10B.

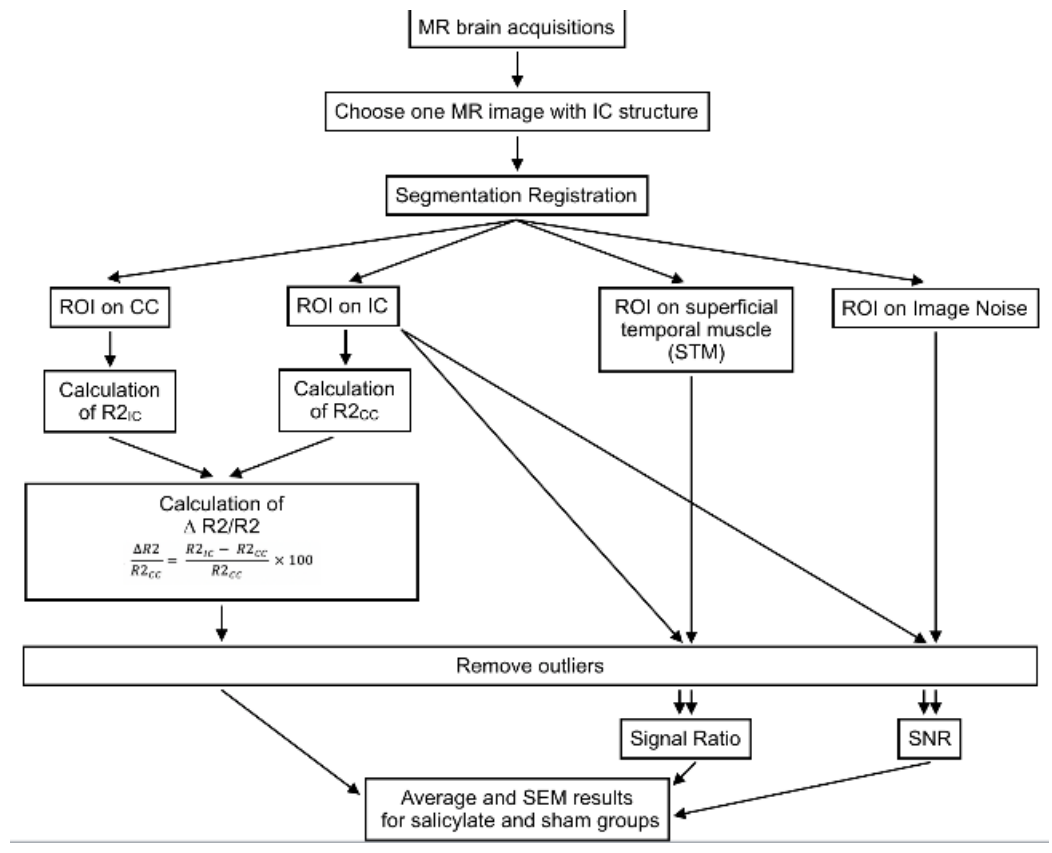

Fig. 9 Schematic representation of MR images processing protocol for IC, CC, image noise, and STM ROIs extraction. Three different analysis methods are displayed: signal-to-noise ratio (SNR), signal intensity ratio $(\mathrm{SIR})$, and $(\triangle R 2 / R 2)$ method. 


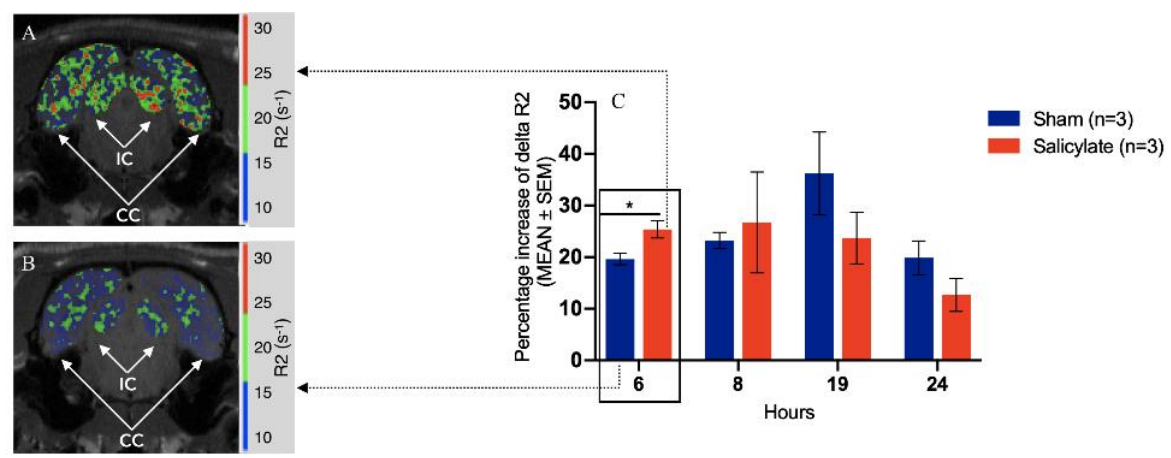

Fig. 10 (a) $R 2$ mapping (RGB colormap) superimposed over $T 1$-weighted MR images on IC and CC of both sides of image. MR images were acquired $6 \mathrm{~h}$ after intraperitoneal Mn2+ injection for salicylate induced tinnitus rat. (b) MR image $6 \mathrm{~h}$ after administration of IP administration of $\mathrm{MnCl} 2$ for sham rat without treatment. (c) Measurements $\Delta R 2 / R 2$ in IC at $6,8,19$, and $24 \mathrm{~h}$ after $\mathrm{MnCl} 2$ administration.

$* p<0.05$

To rule out inter-individual $\mathrm{MnCl}_{2}$ diffusion problems especially by the TT route [44] the percentage increase of $\Delta \mathrm{R} 2 / \mathrm{R} 2$ was calculated from the $\mathrm{R} 2$ relaxation rate on the $\mathrm{IC}$ and $\mathrm{CC}$ according to the following equation:

$$
\frac{\Delta R 2}{R 2_{C C}}=\frac{R 2_{I C}-R 2_{C C}}{R 2_{C C}} \times 100
$$

Figure 11 presents an example of the distribution of $\Delta R 2 / R 2$ values for both groups (sham and salicylate) showing the typical and atypical $\Delta \mathrm{R} 2 / \mathrm{R} 2$ values to remove outliers that are far from the main part of the distribution. Finally, the SNR and SIR analyses were performed on the selected animals.

\section{Statistical analysis}

Descriptive statistics by groups are provided as the mean \pm SEM (arithmetic mean \pm Standard Error of the Mean) for SNR quantification, SIR and percentage increase using the $\Delta R 2$ methods. Statistical significance was determined using Student's $t$-test using Prism software with the following significance value: $* \mathrm{p}<0.05, * * \mathrm{p}<0.01$ and $* * * \mathrm{p}<0.001 ; \mathrm{n}$ represents the number of independent experiments. 


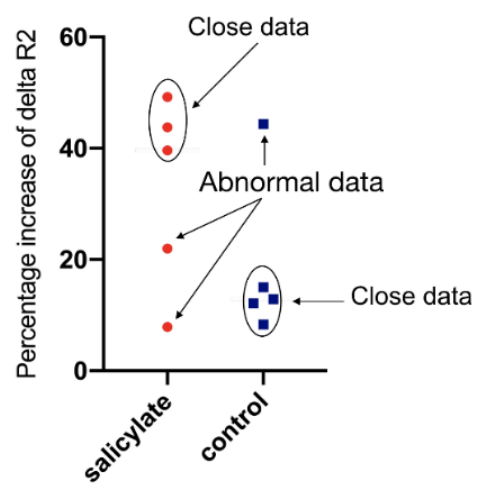

Fig. 11 Example showing the distribution of $\Delta R 2 / R 2$ values for salicylate and sham groups. Data too far from all data in the group (close data) were removed.
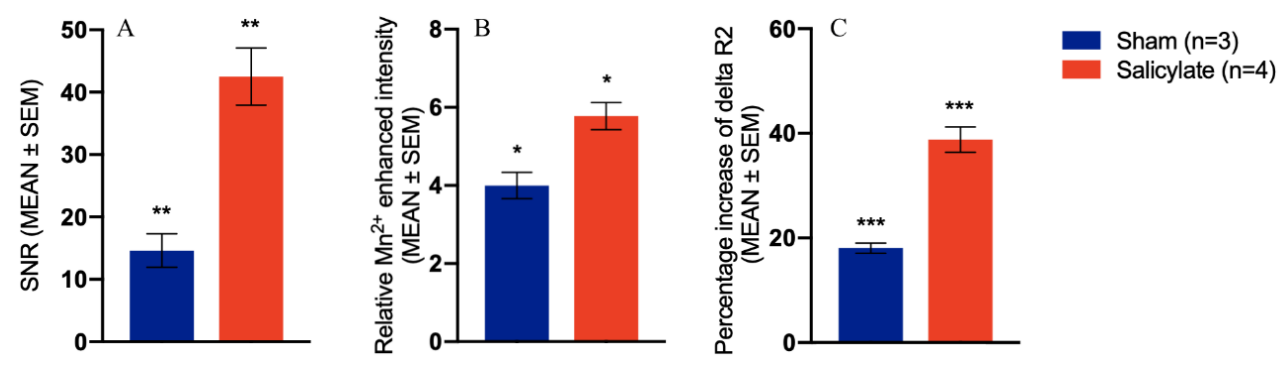

Fig. 12 Comparison between salicylate-treated group and sham group with SNR analysis method (a), SIR method (b), and $\Delta R 2 / R 2$ method (c). $* p<0.005, * * p<0.01, * * * p<0.001$.

\subsection{RESULTS}

\section{Series A}

For the series A experiments, rats received $20 \mu \mathrm{L}$ of $\mathrm{MnCl}_{2}$ by the TT route 24 hours before the MRI session. The SIT group received sodium salicylate $(300 \mathrm{mg} / \mathrm{kg}$, i.p.) once a day from D1 to D4 and the sham group was injected with a similar volume of saline solution. The ipsilateral IC showed an uptake of manganese after salicylate exposure when the contrast agent was injected 24 hours prior to imaging and when the last dose of sodium salicylate was injected 18 hours before MEMRI. As shown in Figure 12, a significant increase of SIR, SNR and $\Delta R 2 / R 2$ was observed in the SIT group. These results confirmed an increase of neural activity in the IC under salicylate treatment using a drug dose known to consistently induced tinnitus [7, 43, 48, 51]. A lower increase was observed when the MR images were analyzed with the SIR method compared to $\Delta \mathrm{R} 2 / \mathrm{R} 2$ and SNR methods.

In addition, SNR results showed the largest mean difference between both groups (sham and salicylate), but we observed less variability of statistical significance with the $\Delta R 2 / R 2$ analysis method. 


\section{Series B}

All animals injected with $\mathrm{MnCl}_{2}$ by intraperitoneal administration at 6, 8, 19 or 24 hours prior to imaging did not exhibit significant differences in MEMRI results with the SNR and SIR analysis methods ( $p>0.05$ ). With the $\Delta R 2 / R 2$ analysis method, the group that received an injection at 6 hours before the MEMRI showed a significant difference between the salicylate treated group and the sham group ( $\mathrm{p}=0.047$ ) (figure 10.C). The $\Delta \mathrm{R} 2 / \mathrm{R} 2$ mappings in figure 10.A and 10.B revealed higher $\Delta \mathrm{R} 2 / \mathrm{R} 2$ values on ROIs for the IC and $\mathrm{CC}$ for the salicylateinduced tinnitus group compared to the sham group.

\section{Series C}

To validate MEMRI analysis methods and confirm the presence of tinnitus, we added the GPIAS behavior data for the same animals. In Figure 13, GPIAS was applied at baseline and 2 hours after the first salicylate administration. The rats, which fulfilled the inclusion criteria at baseline measures (with $\mathrm{ABR} \leq 40 \mathrm{~dB}$ at $16 \mathrm{kHz}$ in both ears, with a \% of PPI inhibition at BBN $\square 50 \%$ and with a \% of Gap inhibition at BBN $\square 20 \%$ ), were selected and distributed to the two groups. Exclusions of animals from the analysis were performed based on three predefined criteria:

- The time of response to the pulse: from 265 to $320 \mathrm{~ms}$

- The percentage of Gap inhibition $<20 \%$ at baseline for all groups

- The percentage of PPI inhibition (previously defined) $<50 \%$ at baseline for all groups As a reminder, for all animals at baseline, the startle reflex with gap insertion (Gap) was expected to be significantly lower than without the gap (No Gap) because the gap was assumed to be perceptible in the absence of tinnitus. In contrast, the animals with putative tinnitus that presumably have tinnitus should have startle reflexes similar to the No Gap condition because the tinnitus fills in the silent gap. For the sham group, the nonresponsive animals at baseline (who have weak Gap responses) were excluded for the concerned frequency. At baseline, all groups presented a similar \% of Gap inhibition. The Sham group had a constant \% of Gap inhibition at D1. As expected, a decrease of the $\%$ of Gap inhibition was observed in the Salicylate group compared to the Sham group. After analysis of GPIAS results, 6 rats were selected based on the criteria described above to perform MEMRI on 3 animals per group: 3 salicylate-treated rats (Figure 13A) and 3 sham rats (Figure 13B).

At day 2, all animals were injected with $\mathrm{MnCl}_{2}$ (i.p) 6 hours before and then with either salicylate (experimental group) or vehicle (control group) 4 hours before MEMRI measures. No significant differences in manganese uptakes were found with SNR and SIR results with our experimental protocol. In accordance with GPIAS results, $\Delta \mathrm{R} 2 / \mathrm{R} 2$ showed a significant difference between groups confirming the presence of tinnitus in the salicylate treated animals in Figure 13C. 

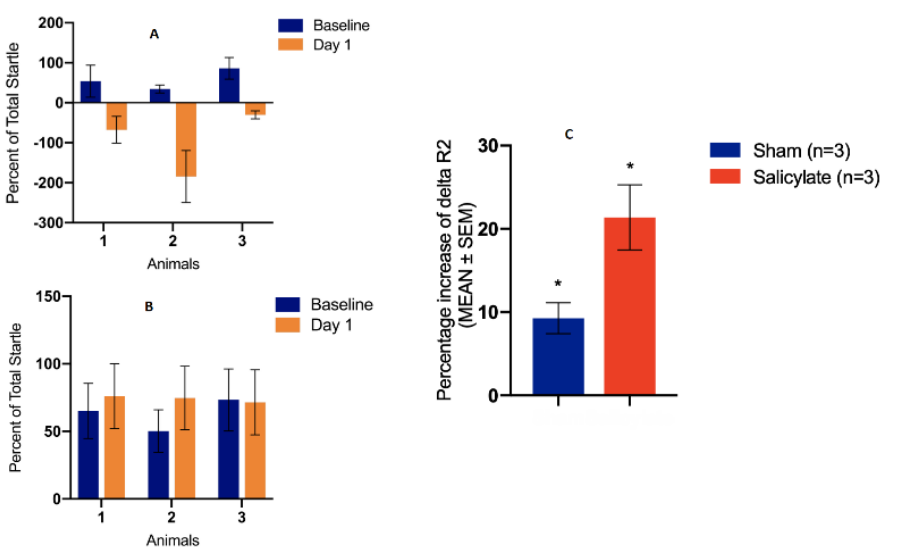

Fig. 13 Relationship between GAP data and Delta $R 2$ method. (a) GAP detection before and after sodium salicylate administration $(300 \mathrm{mg} / \mathrm{kg}$ ) for three salicylate-treated rats. After collecting the GAP data, the sham and salicylate-treated rats were assessed with MEMRI. (b) GAP detection during baseline and day 1 for three sham rats. (c) Percentage increase of delta $R 2$ between sham group and salicylate-induced tinnitus group.

\subsection{DISCUSSION}

In the literature, an enhancement of the $1 \mathrm{H}$ NMR signal in the presence of $\mathrm{Mn} 2+$ in the auditory pathway associated with noise or salicylate-induced tinnitus was observed [53]. To our knowledge, we report for the first time the use of three distinct MEMRI analysis methods to identify significant changes in Mn2+ on the same sham and salicylate-treated rats. If SNR and SIR analysis methods are well-established methods [7, 35, 43, 44, 54], the $\Delta \mathrm{R} 2 / \mathrm{R} 2$ method is an innovative approach based on the NMR relaxation rate R2. In our case, the three MEMRI analysis methods were compared on the same tinnitus animal model (SIT model) in order to determine the optimal processing protocol to apply.

\section{Comparison Between Three MEMRI Analysis Methods}

To achieve our analysis data on the different experimental protocols (Figure 10 and Figure 12) in the first step the $\Delta \mathrm{R} 2 / \mathrm{R} 2$ method was applied enabling to determine a selection of animals after removing outliers $\Delta R 2 / R 2$ values (example in Figure 11). All rats with atypical $\Delta R 2 / R 2$ values in IC were excluded from the animal cohort. Thus, SNR and SIR were then carried out. Figure 12 presents data of MEMRI analysis methods from MRI acquisitions of the rat brain 24 hours after $\mathrm{MnCl}_{2}$ TT administration on a SIT model. Significant increases of $\mathrm{Mn}^{2+}$ signal intensities were observed in each analysis method. However, we observed lower variability and larger statistically significant values with the $\Delta \mathrm{R} 2 / \mathrm{R} 2$ analysis method. This new method is based on an NMR relaxation rate (R2) and is proportional to the $\mathrm{MnCl}_{2}$ concentration as shown in following equation:

$$
R i=R i_{0}+r_{i}\left(\left[\mathrm{MnCl}_{2}\right]_{\text {diffusion }}+\left[\mathrm{MnCl}_{2}\right]_{\text {tinnitus }}\right) ; i=1,2
$$


From the work of Caravan and colleagues [54], we estimated the relaxivity ( $\mathrm{r} 1=5.14 \mathrm{mM}-1$; $\mathrm{r} 2=117 \mathrm{mM}-1)$ and found agreement with their data. The $\Delta \mathrm{R} 2 / \mathrm{R} 2$ method relies on the NMR transversal relaxation rate (R2) changes while SNR and SIR are based on longitudinal relaxation rate R1 contrasts from the signal intensities in IC and cerebral cortex (CC). In our study, we observed that the amount of $\mathrm{Mn}^{2+}$ uptake into the brain can vary between rats, particularly during TT administration making the SNR and SIR analysis difficult to perform. This inter-individual diffusion of $\mathrm{MnCl}_{2}$ is corrected in the $\Delta \mathrm{R} 2 / \mathrm{R} 2$ approach between the IC and CC. In series A, for the rest of the statistical analysis three salicylate-treated rats and two sham rats were excluded because of atypical $\Delta \mathrm{R} 2 / \mathrm{R} 2$ values. It has been shown in the literature that strong enhancement of signal intensity after $\mathrm{MnCl}_{2}$ administration was observed in some non-auditory areas like the hippocampus $[8,35,37]$. Preliminary data demonstrated for two SIT excluded rats that the mean relaxation rate (R2) in hippocampus were clearly similar to mean $\mathrm{R} 2$ values in the hippocampus of rats which received no $\mathrm{MnCl}_{2}$ or salicylate. Based on these results, it appears that there is considerably variability in $\mathrm{MnCl}_{2}$ administration and uptake. For the last SIT rat excluded, it may be that the rat did not react to the sodium salicylate drug.

Comparing all analysis techniques, the $\Delta \mathrm{R} 2 / \mathrm{R} 2$ method shows less variable results in the SIT and sham groups and can provide robust results compared to the SNR and SIR methods. This $\Delta \mathrm{R} 2 / \mathrm{R} 2$ method is based on a semi-automatic technique decreasing human bias and reducing potential human errors. In addition, $\mathrm{R} 2$ is proportional to the $\mathrm{MnCl}_{2}$ concentration, so the $\Delta \mathrm{R} 2 / \mathrm{R} 2$ method could in principle quantify the presence of $\mathrm{MnCl}_{2}$ and identifying hyperactivity due to tinnitus with a salicylate-induced tinnitus model.

\section{Transtympanic (TT) vs Intraperitoneal (IP) $\mathrm{Mn}^{2+}$ Administration}

In the SIT model, TT and IP administration of $\mathrm{MnCl}_{2}$ revealed significant differences between the salicylate treated and sham groups. Unfortunately, the local $\mathrm{MnCl}_{2}$ administration has some negative consequences in terms of animal wellness with a large variability of $\mathrm{MnCl}_{2}$ diffusion into the cochlea. As previously discussed this could result from position of the needle during TT injections [44]. Afterwards, we tested IP administration at several injection times in order to limit side effects. From the MEMRI study and post processing analysis, we obtained significant results only from the $\Delta \mathrm{R} 2 / \mathrm{R} 2$ method at 6-hours post-treatment (Figure 10.C). It has to be noted that no animal was excluded using $\Delta \mathrm{R} 2 / \mathrm{R} 2$ method. Conversely, for $T T$ administration, significant results were obtained with the different analysis methods. Moreover, the SIT results with the percentage increase of $\Delta \mathrm{R} 2 / \mathrm{R} 2$ was higher with the TT administration than IP administration (Figure 12.C and 10; $<<0,001$ vs. $p<0,05$ ). IP administration provides a lower and bilaterally homogeneous concentration of $\mathrm{Mn} 2+$ compared to TT administration. 


\section{GPAIS vS. MEMRI}

We compared our GPIAS with MEMRI results obtained by IP administration. Despite a less significant difference with $\triangle \mathrm{R} 2 / \mathrm{R} 2$ analysis method obtained with $\mathrm{MnCl}_{2}$ administration (IP) at 6 hours before MEMRI measures, our GPIAS results indicative of tinnitus were associated with an increase in $\triangle \mathrm{R} 2 / \mathrm{R} 2$ in the IC suggesting that this MEMRI methods may be adequate to detect a tinnitus-like neural signature with a salicylate-induced tinnitus model (Figure 13). 


\section{CONCLUSION}

In summary, compared to the SNR and SIR analytical methods, the delta $\Delta R 2 / R 2$ method shows less variability and higher level of statistical significance when assessing $\mathrm{Mn}^{+2}$ in the IC when comparing salicylate-treated rats with sham. The method is based on a semi-automatic segmentation of the brain that reduces experimental bias and increases consistency thereby eliminating operator-dependent issues. The $\mathrm{R} 2$ relaxation rate is proportional to $\mathrm{MnCl}_{2}$ concentration which makes it sensitive and quantitative to the accumulation of $\mathrm{Mn}^{2+}$. Therefore, this new innovative method of quantification is potentially more robust and reproducible compared to other MEMRI analysis methods. If one assumes that $\mathrm{Mn}^{+2}$ uptake in the IC is a reliable metric of tinnitus with all forms of this disorder, then the $\Delta \mathrm{R} 2 / \mathrm{R} 2$ method could conceivably be used to evaluate the effectiveness of some medicines to reduce tinnitus. 


\section{References}

1. Cunningham, L.L., and Tucci, D.L. (2017). Hearing Loss in Adults. N Engl J Med 377, 2465-2473.

2. Wilson, B.S., Tucci, D.L., Merson, M.H., and O'Donoghue, G.M. (2017). Global hearing health care: new findings and perspectives. Lancet 390, 2503-2515.

3. Hina Sultana1, N.M., Tayyaba Dawood3 (2018). TYPE AND DEGREE OF HEARING LOSS IN PATIENTS WITH TINNITUS.

4. Basile, C.E., Fournier, P., Hutchins, S., and Hebert, S. (2013). Psychoacoustic assessment to improve tinnitus diagnosis. PLoS One 8, e82995.

5. Stolzberg, D., Salvi, R.J., and Allman, B.L. (2012). Salicylate toxicity model of tinnitus. Front Syst Neurosci 6, 28.

6. Brozoski, T.J., Ciobanu, L., and Bauer, C.A. (2007). Central neural activity in rats with tinnitus evaluated with manganese-enhanced magnetic resonance imaging (MEMRI). Hear Res 228, 168179.

7. Holt, A.G., Bissig, D., Mirza, N., Rajah, G., and Berkowitz, B. (2010). Evidence of key tinnitusrelated brain regions documented by a unique combination of manganese-enhanced MRI and acoustic startle reflex testing. PLoS One 5, e14260.

8. Yu, X., Wadghiri, Y.Z., Sanes, D.H., and Turnbull, D.H. (2005). In vivo auditory brain mapping in mice with Mn-enhanced MRI. Nat Neurosci 8, 961-968.

9. Chen, Y.C., Li, X., Liu, L., Wang, J., Lu, C.Q., Yang, M., Jiao, Y., Zang, F.C., Radziwon, K., Chen, G.D., et al. (2015). Tinnitus and hyperacusis involve hyperactivity and enhanced connectivity in auditory-limbic-arousal-cerebellar network. Elife 4, e06576.

10. Harding, G.W., and Bohne, B.A. (2009). Relation of focal hair-cell lesions to noise-exposure parameters from a $4-$ or a $0.5-\mathrm{kHz}$ octave band of noise. Hear Res 254, 54-63.

11. Harding, G.W., Bohne, B.A., and Ahmad, M. (2002). DPOAE level shifts and ABR threshold shifts compared to detailed analysis of histopathological damage from noise. Hear Res 174, 158-171.

12. Gauvin, D.V., Yoder, J., Koch, A., Zimmermann, Z.J., and Tapp, R.L. (2017). Down for the count: The critical endpoint in ototoxicity remains the cytocochleogram. J Pharmacol Toxicol Methods 88, 123-129.

13. Viberg, A., and Canlon, B. (2004). The guide to plotting a cochleogram. Hear Res 197, 1-10.

14. Neal, C., Kennon-McGill, S., Freemyer, A., Shum, A., Staecker, H., and Durham, D. (2015). Hair cell counts in a rat model of sound damage: Effects of tissue preparation \& identification of regions of hair cell loss. Hear Res 328, 120-132.

15. Bohne, B.A. (1972). Location of small cochlear lesions by phase contrast microscopy prior to thin sectioning. Laryngoscope $82,1-16$.

16. Hardie, N.A., MacDonald, G., and Rubel, E.W. (2004). A new method for imaging and 3D reconstruction of mammalian cochlea by fluorescent confocal microscopy. Brain research 1000, 200-210.

17. Barbara A. Bohne, G.W.H. (2011). MICROSCOPIC ANATOMY OF THE MO USE INNER EAR

18. Militchin, V., Bohne, B., and W Harding, G. (2011). Mouse Cochlear Dissection Movie.

19. Keithley, E.M., and Feldman, M.L. (1982). Hair cell counts in an age-graded series of rat cochleas. Hear Res 8, 249-262.

20. Spoendlin, H., and Brun, J.P. (1974). The block-surface technique for evaluation of cochlear pathology. Arch Otorhinolaryngol 208, 137-145.

21. Akil, O., Seal, R.P., Burke, K., Wang, C., Alemi, A., During, M., Edwards, R.H., and Lustig, L.R. (2012). Restoration of hearing in the VGLUT3 knockout mouse using virally mediated gene therapy. Neuron 75, 283-293. 
22. Bohne, B.A., Kimlinger, M., and Harding, G.W. (2017). Time course of organ of Corti degeneration after noise exposure. Hear Res 344, 158-169.

23. Chen, G.D., Decker, B., Krishnan Muthaiah, V.P., Sheppard, A., and Salvi, R. (2014). Prolonged noise exposure-induced auditory threshold shifts in rats. Hear Res 317, 1-8.

24. Takada, Y., Takada, T., Lee, M.Y., Swiderski, D.L., Kabara, L.L., Dolan, D.F., and Raphael, Y. (2015). Ototoxicity-induced loss of hearing and inner hair cells is attenuated by HSP70 gene transfer. Mol Ther Methods Clin Dev 2, 15019.

25. Akil, O., and Lustig, L.R. (2013). Mouse Cochlear Whole Mount Immunofluorescence. Bio Protoc 3 .

26. Sun, K.H.X.Z.S.R.J. (2016). Deep Residual Learning for Image Recognition.

27. Yang, S.J.P.a.Q. (2009). A Survey on Transfer Learning

28. Monro, H.R.S. (1951). A Stochastic Approximation Method.

29. Ding, D., Jiang, H., Chen, G.D., Longo-Guess, C., Muthaiah, V.P., Tian, C., Sheppard, A., Salvi, R., and Johnson, K.R. (2016). N-acetyl-cysteine prevents age-related hearing loss and the progressive loss of inner hair cells in gamma-glutamyl transferase 1 deficient mice. Aging (Albany NY) 8, 730-750.

30. Sanz, L., Murillo-Cuesta, S., Cobo, P., Cediel-Algovia, R., Contreras, J., Rivera, T., Varela-Nieto, I., and Avendano, C. (2015). Swept-sine noise-induced damage as a hearing loss model for preclinical assays. Front Aging Neurosci 7, 7.

31. Lockwood, A.H., Salvi, R.J., and Burkard, R.F. (2002). Tinnitus. N Engl J Med 347, 904-910.

32. Jastreboff, P.J., Brennan, J.F., Coleman, J.K., and Sasaki, C.T. (1988). Phantom auditory sensation in rats: an animal model for tinnitus. Behav Neurosci 102, 811-822.

33. Boyen, K., Baskent, D., and van Dijk, P. (2015). The Gap Detection Test: Can It Be Used to Diagnose Tinnitus? Ear Hear 36, e138-145.

34. Turner, J.G., Brozoski, T.J., Bauer, C.A., Parrish, J.L., Myers, K., Hughes, L.F., and Caspary, D.M. (2006). Gap detection deficits in rats with tinnitus: a potential novel screening tool. Behav Neurosci $120,188-195$.

35. Muca, A., Standafer, E., Apawu, A.K., Ahmad, F., Ghoddoussi, F., Hali, M., Warila, J., Berkowitz, B.A., and Holt, A.G. (2018). Tinnitus and temporary hearing loss result in differential noiseinduced spatial reorganization of brain activity. Brain structure \& function 223, 2343-2360.

36. Galazyuk, A., and Hebert, S. (2015). Gap-Prepulse Inhibition of the Acoustic Startle Reflex (GPIAS) for Tinnitus Assessment: Current Status and Future Directions. Front Neurol 6, 88.

37. Lee, J.W., Park, J.A., Lee, J.J., Bae, S.J., Lee, S.H., Jung, J.C., Kim, M.N., Lee, J., Woo, S., and Chang, Y. (2007). Manganese-enhanced auditory tract-tracing MRI with cochlear injection. Magn Reson Imaging 25, 652-656.

38. Pautler, R.G. (2004). In vivo, trans-synaptic tract-tracing utilizing manganese-enhanced magnetic resonance imaging (MEMRI). NMR Biomed 17, 595-601.

39. Cheung, S.W., Nagarajan, S.S., Bedenbaugh, P.H., Schreiner, C.E., Wang, X., and Wong, A. (2001). Auditory cortical neuron response differences under isoflurane versus pentobarbital anesthesia. Hear Res 156, 115-127.

40. Malheiros, J.M., Paiva, F.F., Longo, B.M., Hamani, C., and Covolan, L. (2015). ManganeseEnhanced MRI: Biological Applications in Neuroscience. Front Neurol 6, 161.

41. Schumacher, J.W., Schneider, D.M., and Woolley, S.M. (2011). Anesthetic state modulates excitability but not spectral tuning or neural discrimination in single auditory midbrain neurons. $\mathbf{J}$ Neurophysiol 106, 500-514.

42. Ter-Mikaelian, M., Sanes, D.H., and Semple, M.N. (2007). Transformation of temporal properties between auditory midbrain and cortex in the awake Mongolian gerbil. J Neurosci 27, 6091-6102.

43. Jin, S.U., Lee, J.J., Hong, K.S., Han, M., Park, J.W., Lee, H.J., Lee, S., Lee, K.Y., Shin, K.M., Cho, J.H., et al. (2013). Intratympanic manganese administration revealed sound intensity and frequency dependent functional activity in rat auditory pathway. Magn Reson Imaging 31, 1143-1149. 
44. Lee, H.J., Yoo, S.J., Lee, S., Song, H.J., Huh, M.I., Jin, S.U., Lee, K.Y., Lee, J., Cho, J.H., and Chang, Y. (2012). Functional activity mapping of rat auditory pathway after intratympanic manganese administration. Neuroimage 60, 1046-1054.

45. Paul, A.K., Lobarinas, E., Simmons, R., Wack, D., Luisi, J.C., Spernyak, J., Mazurchuk, R., AbdelNabi, H., and Salvi, R. (2009). Metabolic imaging of rat brain during pharmacologically-induced tinnitus. Neuroimage 44, 312-318.

46. Rancz, E.A., Moya, J., Drawitsch, F., Brichta, A.M., Canals, S., and Margrie, T.W. (2015). Widespread vestibular activation of the rodent cortex. J Neurosci 35, 5926-5934.

47. Silva, A.C., and Bock, N.A. (2008). Manganese-enhanced MRI: an exceptional tool in translational neuroimaging. Schizophr Bull 34, 595-604.

48. Groschel, M., Gotze, R., Muller, S., Ernst, A., and Basta, D. (2016). Central Nervous Activity upon Systemic Salicylate Application in Animals with Kanamycin-Induced Hearing Loss--A Manganese-Enhanced MRI (MEMRI) Study. PLoS One 11, e0153386.

49. Jung, D.J., Han, M., Jin, S.U., Lee, S.H., Park, I., Cho, H.J., Kwon, T.J., Lee, H.J., Cho, J.H., Lee, K.Y., et al. (2014). Functional mapping of the auditory tract in rodent tinnitus model using manganese-enhanced magnetic resonance imaging. Neuroimage 100, 642-649.

50. Liu, Y., Li, X., Ma, C., Liu, J., and Lu, H. (2005). Salicylate blocks L-type calcium channels in rat inferior colliculus neurons. Hear Res 205, 271-276.

51. Hu, S.S., Mei, L., Chen, J.Y., Huang, Z.W., and Wu, H. (2014). Expression of immediate-early genes in the inferior colliculus and auditory cortex in salicylate-induced tinnitus in rat. Eur $\mathbf{J}$ Histochem 58, 2294.

52. Spivak, M., Weston, J., Bottou, L., Kall, L., and Noble, W.S. (2009). Improvements to the percolator algorithm for Peptide identification from shotgun proteomics data sets. J Proteome Res 8, 3737-3745.

53. Bauer, C.A., Brozoski, T.J., Rojas, R., Boley, J., and Wyder, M. (1999). Behavioral model of chronic tinnitus in rats. Otolaryngol Head Neck Surg 121, 457-462.

54. Caravan, P., Farrar, C.T., Frullano, L., and Uppal, R. (2009). Influence of molecular parameters and increasing magnetic field strength on relaxivity of gadolinium- and manganese-based T1 contrast agents. Contrast Media Mol Imaging 4, 89-100. 


\section{Figure Legends:}

Figure 1: Precision-Sensitivity curves for IHC and OHC objects on the test subregions. Note the lower overall performance for IHCs is due to the bias of over sampling of OHCs.

Figure 2: Time in minutes for annotating each fragment among human users compared to the algorithm.

Figure 3: Box-Whisker (median $\pm S D$ ) distributions for $\%$ variability of counts for manual counting.

Figure 4: Box-Whisker plots (median $\pm S D$ ) for the sensitivity and precision scores for the algorithm versus test cases.

Figure 5: Example comparing cochleograms obtained from human manual (orange) countinb and automated (blue) counting of OHCs and ICHs in cisplatin treated rats. Solid line is the mean with bands for the standard deviation.

Figure 6: Image of cochlear fragment where only a single row of IHCs (red dots) is annotated.

Figure 7: Schematics of three different experimental protocols used in the study with $\mathrm{MnCl}_{2}$ in which salicylate was administered by $(A)$ transtympanic administration $(A)$ and by $(B, C)$ intraperitoneal route.

Figure 8: Tl-weighted MR coronal brain slice acquired with 9.4 T scanner (400 MHz). Arrows point to examined regions of interest (ROIs): Image Noise, Superficial Temporal Muscle (STM), Inferior Colliculus (auditory structure), Cerebral Cortex (non-auditory structure).

Figure 9: Schematic representation of MR images processing protocol for IC, CC, image noise and STM ROIs, extraction. Three different analysis methods are displayed: Signal to noise ratio (SNR), Signal Intensity Ratio (SIR) and ( $\triangle R 2 / R 2)$ method.

Figure 10: (A) R2 mapping (RGB colormap) superimposed over T1-weighted MR images on IC and CC of both sides of image. MR images were acquired 6 hours after intraperitoneal $\mathrm{Mn}^{2+}$ injection for salicylate induced tinnitus rat. (B) MR image 6 hour after administration of IP administration of $\mathrm{MnCl}_{2}$ for sham rat without treatment. (C) Measurements $\triangle R 2 / R 2$ in IC at 6, 8, 19 and $24 \mathrm{~h}$ after $\mathrm{MnCl}_{2}$ administration. $* p<0.05$.

Figure 11: Example showing the distribution of $\Delta R 2 / R 2$ values for salicylate and sham groups. Data too far from all data in the group (close data) were removed.

Figure 12: Comparison between salicylate-treated group and sham group with SNR analysis method (A), SIR method (B) and $\triangle R 2 / R 2$ method $(C)$. *p $<0,005, * * p<0,01$, ***p<0,001. 
Figure 13: Relationship between GAP data and Delta R2 method. (A) GAP detection before and after sodium salicylate administration $(300 \mathrm{mg} / \mathrm{kg})$ for three salicylate-treated rats. After collecting the GAP data, the sham and salicylate-treated rats were assessed with MEMRI. (B) GAP detection during baseline and day 1 for three sham rats. $(C)$. Percentage increase of delta $R 2$ between sham group and salicylateinduced tinnitus group. 